cancer. In HIV-negative individuals, pooled HPV prevalence was $6.42 \% \quad(95 \%$ CI: $4.13-9.18 \%)$ for normal cytology, 18.86\% (95\% CI: 10.84-28.49\%) for head and neck lesions, and $28.86 \%$ (95\% CI: 24.26-33.70\%) for head and neck cancer. Pooled HPV 16 prevalence increased with diagnosis severity, from $2.14 \%$ (95\% CI: $0.86-3.94 \%$ ) in normal cytology to $20.47 \%$ (95\% CI: $16.58-24.65 \%)$ in head and neck cancer in HIV negative individuals. HPV prevalence varied by diagnosis, subsite, region, but not by gender. Data for HIVpositive patients were too limited to analyze.

Conclusion In HIV negative individuals, HPV prevalence increased with diagnosis severity. And HPV 16 was the most carcinogenic HPV type in head and neck, with enrichment from normal cytology to head and neck cancer.

Disclosure No significant relationships.

\section{P830 FEASIBILITY OF AN ONLINE HPV SELF-COLLECTION SCREENING PROGRAM IN CANADA: DIGITAL HEALTH LITERACY IN SOUTH ASIAN WOMEN}

${ }^{1}$ Sandy Zhang*, ${ }^{2}$ Heather Pedersen, ${ }^{3}$ Laurie Smith, ${ }^{2} \mathrm{C}$ Sarai Racey, ${ }^{3}$ Dirk Van Niekerk, ${ }^{3}$ Marette Lee, ${ }^{4}$ Mark Gilbert, ${ }^{4}$ Devon Haag, ${ }^{2}$ Gina Ogilvie. 'Simon Fraser University, Burnaby, Canada; ${ }^{2}$ University of British Columbia, Vancouver, Canada; ${ }^{3} B C$ Cancer, Vancouver, Canada; ${ }^{4} B C$ Centre for Disease Control, Clinical Prevention Services, Vancouver, Canada

\subsection{6/sextrans-2019-sti.875}

Background Women who do not regularly attend cervical cancer screening are at increased risk for cervical cancer. In British Columbia (BC), approximately 30\% of women aged 21-69 years are under-screened. As cervical cancer screening in BC moves towards the use of primary HPV testing, there is an opportunity to address screening barriers women face through self-collected, rather than clinician collected specimens. CervixCheck is an internet-based program for HPV self-collection being piloted in communities across BC with low screening rates. To inform the implementation of CervixCheck, we investigated digital health literacy (DHL) in South Asian women.

Methods A cross-sectional anonymous survey was administered July-August 2018 through collaborating primary care clinics in predominantly South Asian communities in the Fraser Health Region of BC. The study population was a convenience sample of women 30-65 years of age, presenting at a primary care clinic. Women were administered the survey on a tablet, which collected demographic, screening history, and internet use information. DHL was measured using the validated eHEALS and Digital Health Literacy Instruments.

Results 51 women participated from four family practices where $30 \%$ of women were 50 years or older. $29.4 \%$ of women self-reported not having had a Pap test in the last 3 years. English (86\%) and Punjabi (58\%) were the most common languages participants reported reading and speaking. Majority of women reported using the internet daily $(82.4 \%)$, with mobile phones being the most common device $(72.6 \%)$. DHL was higher in under-screened women. Over $80 \%$ of women responded that they would be likely to very likely to participate in self-collected screening using CervixCheck.

Conclusion The survey revealed CervixCheck is a promising digital health platform to increase cervical cancer screening uptake among under-screened South Asian women. Findings were used to inform CervixCheck website design and program resources in preparation for its launch.
Disclosure No significant relationships.

\section{P831 CHARACTERISTICS ASSOCIATED WITH HPV-RELATED EXTERNAL GENITAL LESIONS AMONG YOUNG ADULTS IN BRAZIL}

${ }^{1} J u l i a n a$ Comerlato, ${ }^{2}$ Marina Bessel ${ }^{*}$, ${ }^{2}$ Natalia Kops, ${ }^{2}$ Jaqueline Horvath, ${ }^{3}$ Bruna Vieira Fernandes, ${ }^{3}$ Antonella Jacobsen, ${ }^{3}$ Eliana Wendland. ${ }^{1}$ Moinhos de Vento Hospital, Porto Alegre, Brazil; ${ }^{2}$ Hospital Moinhos de Vento, Porto Alegre, Brazil; ${ }^{3}$ Universidade Federal de Ciências da Saúde de Porto Alegre, Porto Alegre, Brazil

\subsection{6/sextrans-2019-sti.876}

Background HPV-related external genital lesions are the most common outcome of HPV infection, a sexual transmitted disease responsible by low-grade squamous intraepithelial lesion (LSIL) and $99 \%$ of cervical cancer. The aim of this study was to assess the prevalence of HPV-related lesions and associated factors in the Brazilian young adult population.

Methods We included 7,694 unvaccinated people from 16 to 25 years recruited from primary care units and submitted to interview, genital examination and cervical/penile sampling. Linear Array ${ }^{\boxplus}$ Test (Roche) was used for HPV detection and genotyping.

Results The prevalence of HPV-related lesions was 4\% (234), being more frequent in men $(5.8 \%)$ than women $(2.3 \%)$. From those, 63\% $(\mathrm{p}<0.03)$ were positive to HPV. The HPV genotyping distribution comprised all the 37 HPV types tested. However, the more frequent types were the 16 (12.5\%), 6 (9.2\%), 11 (8.8\%), 62 (8.7\%), 58 (8.4\%), 52 $(8.2 \%)$ and $51(8 \%)$. Number of sexual partners in the last year $(p=0.0005)$, multiple HPV infection $(p<0.0247)$, smoking $(p=0.04)$, use of hormonal contraceptives $(p=0.04)$ and presence of other STIs $(\mathrm{p}<0.0001)$ were associated with the presence of lesion.

Conclusion We found a high prevalence of genital lesion in this young adult population. As expected, most participants were HPV positive. Although HPV 16 was not usually related to external lesions, it was the most frequent HPV type found followed by HPV 6 and 11. Sexual behavior, smoking and hormonal contraception were the main factors associated with genital lesions. Increase awareness about healthy sexual behaviors and immunization coverage should be used as a target to prevent HPV infection and related lesions in the young Brazilian population.

Disclosure No significant relationships.

\section{P832 EFFECTIVENESS OF THE QUADRIVALENT HPV VACCINE AGAINST HSIL AND CIN: A DATA-LINKAGE STUDY}

${ }^{1}$ Robine Donken*, ${ }^{2}$ Arianne Albert, ${ }^{1} \mathrm{C}$ Sarai Racey, ${ }^{3}$ Laurie Smith, ${ }^{3}$ Dirk Van Niekerk, ${ }^{3}$ John Spinelli, ${ }^{1}$ Heather Pedersen, ${ }^{4}$ Mel Krajden, ${ }^{5}$ Monika Naus, ${ }^{6}$ Cindy Masaro, ${ }^{6}$ Meena Dawar, ${ }^{7}$ Manish Sadarangani, ${ }^{1}$ Gina Ogilvie. ' University of British Columbia, Vancouver, Canada; ${ }^{2}$ Women's Health Research Institute, Vancouver, Canada; ${ }^{3} B C$ Cancer, Vancouver, Canada; ${ }^{4} B C C D C$, Vancouver, Canada; ${ }^{5}$ University of British Columbia, Faculty of Medicine, Vancouver, Canada; ${ }^{6}$ Vancouver Coastal Health, Vancouver, Canada; ${ }^{7} B C$ Children's Hospital Research Institute, Vaccine Evaluation Center, Vancouver, Canada

\subsection{6/sextrans-2019-sti.877}

Background Although originally approved for three-doses, two doses of the HPV vaccine are now approved for 9-14 year olds in British Columbia (BC), Canada. Post-hoc analyses have shown similar efficacy compared to three-doses even after 